\title{
Facile, productive and cost-effective synthesis of a novel tetrazine-based iron oxide nanoparticle for targeted image contrast agents and nanomedicines
}

\author{
1 Marco M. Meloni • Stephen Barton • Alexandru Chivu • \\ 2 Juan C. Kaski • Wenhui Song • Taigang He \\ 3
}

4 Abstract We have developed an operationally simple, time 5 and cost-effective protocol to produce a novel tetrazine-based 6 iron oxide nanoparticle using commercially available and 7 inexpensive materials. Our protocol proceeds at room 8 temperature and uses Hexafluorophosphate Azabenzotriazole 9 Tetramethyl Uronium, a well-known, widely used reagent for 10 the large-scale industrial production of important 11 pharmaceuticals. The nanoparticles obtained have a diameter 12 range between 16 and $21 \mathrm{~nm}$ and showed no toxicity against 13 endothelial cell lines. The tetrazine moiety on the 14 nanoparticle surface could potentially allow further 15 attachment of specific targeting vectors by using so-called 16 copper-free click chemistry. We therefore anticipate that our 17 protocol can represent a significant breakthrough in the future 18 development and commercialization of improved, tissue19 specific contrast agents and drug delivery for clinical 20 diagnosis, monitoring and therapy of diseases at an 21 asymptomatic stage.

22

23 Keywords Diagnosis • Magnetic Resonance Imaging • Iron 24 Oxide Nanoparticles • Copper-free click Chemistry • 25 Bioconjugation $\bullet$ Synthesis

26

\section{Introduction}

29 Since their discovery (Ohgushi, 1978), Iron Oxide 30 Nanoparticles (IONPs) have emerged as powerful tools for a 31 wide scope of applications such as in the fields of biolabeling, 32 medical diagnostics, and therapy (Colombo et al. 2012). In 33 particular as contrast agents for magnetic resonance imaging 34 (MRI), IONPs have attracted enormous attention. IONPs 35 have a transverse relaxivity which increases at higher 36 magnetic fields, resulting in enhanced signal-to-noise ratio 37 with lower dosages (Mishra et al. 2016). The use of IONPs 38 for diagnostic imaging is therefore expected to increase 39 steadily. Unlike Gd-based contrast agents (CAs), IONPs have 40 proven safe with some systems already approved for clinical 41 use (Schmitz et al. 2001; Trivedi et al. 2004). With the recent 42 advent of molecular imaging (Meloni et al. 2017), IONPs are

Marco M. Meloni • Juan C. Kaski • Taigang He

The Cardiology Academy Group, St George's University of London, Cranmer Terrace, SW17 0RE, London, UK.

Marco M. Meloni • Stephen Barton

School of Pharmacy and Chemistry, Kingston University, Penhryn Road, KT1 2EE, London, UK.

Alexandru Chivu • Wenhui Song

UCL Centre for Biomaterials, Division of surgery \& Interventional Science, University College London, Pond Street, NW3 2PS, London, UK.
43 increasingly used for the preparation of targeted CAs, 44 allowing non-invasive visualization of biomolecules which 45 are the signatures of diseases in specific body tissues, both in 46 vivo and in real time. The preparation of these targeted CAs 47 firstly involves coating of the IONPs surface with a 48 biocompatible, clinically approved polymer, followed by its 49 functionalization with a targeting vector which allows tissue 50 specificity (A. M. Morawski et al. 2005).

51

52 This process, so-called standard bioconjugation, uses well53 established synthetic chemistry and involves the formation of 54 stable chemical bonds. Despite being reliable, this method 55 can lead to low yields and produce toxic byproducts, resulting 56 in expensive, time-consuming purifications and toxicological 57 screenings. A number of years may therefore be required for 58 the translation, large scale production and commercialization 59 of targeted, nanoparticle-based CAs into the clinical market. 60

61 Copper-free click reactions recently emerged as a powerful, 62 time-saving bioconjugation strategy to prepare targeted CAs 63 (Devaraj and Weissleder 2011; McKay and Finn 2014). 64 Copper-free click reactions are operationally simpler and 65 proceed at room temperature. They eliminate the use of 66 cytotoxic copper catalysts (Baskin JM et al. 2007) and are 67 inert to both water and oxygen. They also generate minimal, 68 inoffensive byproducts, providing the best yield with the 69 highest reaction rates. Copper-free click reactions can 70 proceed in living organisms, allowing the targeting of 71 biomolecules with high specificity without altering any native 72 biochemical process. Among the arsenal of available copper73 free click reactions, the Inverse Electron Demand Diels Alder 74 (IEDDA) reaction stands out as the most promising one for 75 its rapid reaction rate and generation of safe, inert nitrogen as 76 the only byproduct (Oliveira et al. 2017). IEDDA reactions 77 also involve tetrazines and cycloalkenes, both of which are 78 easier substrates to prepare if compared to 79 azadibenzocyclooctynes (DIBACs) (Debets et al. 2010) and 80 biarylazacyclooctynones (BARACs) (Kuzmin et al. 2010).

81 Despite such significant advantages, the preparation of 82 targeted, nanoparticle-based CAs using IEDDA is a relatively 83 unexplored field. A possible reason is a shortage of reliable 84 protocols to prepare tetrazine-based IONPs. Therefore, the

Taigang He

Royal Brompton Hospital, Imperial College London, London, UK.

Marco M. Meloni $(\bowtie) \bullet$ Taigang He $(\bowtie)$

The Cardiology Academy Group, St George's University of London, Cranmer Terrace, SW17 0RE, London, UK.

e-mail: mmeloni@sgul.ac.uk

e-mail: the@sgul.ac.uk 
1 identification of novel, green and efficient ways to prepare 2 these functional nanoparticles can accelerate the development 3 of improved contrast agents for diagnosis and therapy. In this 4 paper we designed and synthesized a novel route to a 5 tetrazine-bound nanoparticle 4 (Scheme 1).

6

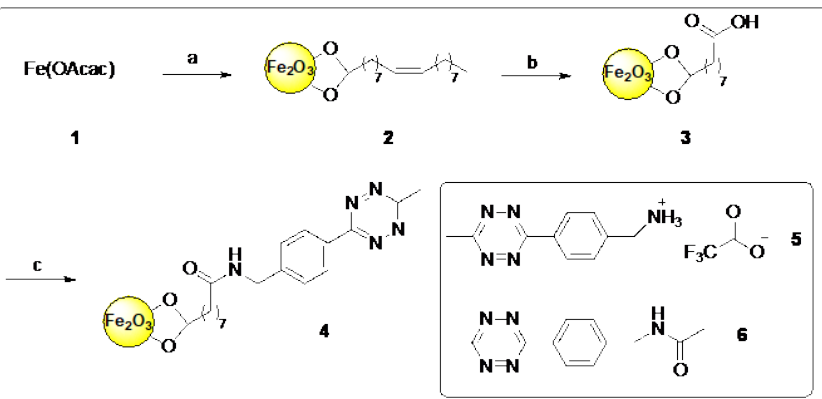

7

8 Scheme 1 (a) Oleic acid, oleylamine, phenyl ether (b) BTAC,

$9 \mathrm{KMnO}_{4}$ (c). Tetrazine 5, HATU, DIPEA, $\mathrm{CH}_{2} \mathrm{Cl}_{2}$, at room 10 temperature.

11

12 As illustrated above, our strategy involved the loading of a 13 tetrazine moiety to nanoparticles 3 using 14 Hexafluorophosphate Azabenzotriazole Tetramethyl 15 Uronium (HATU), a readily available, inexpensive and well16 known reagent. Our overall approach to nanoparticles 4 can 17 be exploited for the future production of improved contrast 18 reagents, with potential application also in the fields of 19 nanomedicine.

20

\section{Results and discussion}

22

23 In this work, we successfully designed and developed an 24 operationally simple, economical and productive approach to 25 produce a novel tetrazine-decorated IONP 4 (Scheme 1, page 26 2). Our protocol is reproducible by taking an advantage of 27 well-established chemistry and usages of building blocks 3 28 and 5, both accessible via established published protocols 29 (Herranz et al. 2008; Evans et al. 2014). Nanoparticles 4 were 30 obtained via coupling between carboxylic acid IONPs 3 and 31 tetrazine-based amine 5 (Scheme 1, page 2). Our method 32 builds upon the reaction between a carboxylic acid and an 33 amine, which produces extremely stable chemical bonds even 34 in a biological environment and is one of the most robust, 35 known reactions in synthetic chemistry (Jaradat, 2018). 36 During this process a challenge also emerged due to the 37 presence of the paramagnetic core both in $\mathbf{3}$ and $\mathbf{4}$, which 38 excluded quantitative reaction monitoring via NMR. 39 Therefore, we first decided to validate the chemistry using 40 acetic acid as a surrogate for nanoparticles 3 . We wanted a 41 fast, high yielding protocol to obtain model compound 6 . 42 After unsuccessful attempts to prepare this latter using $\mathrm{N}$ 43 hydroxysuccinimide (NHS) and 1-Ethyl-3-(344 dimethylaminopropyl)carbodiimide (EDC) in water (Herranz 45 et al. 2008), we found that the system HATU/DIPEA/C $\mathrm{H}_{2} \mathrm{Cl}_{2}$ 46 afforded 6 in 92\% yield after only 3 hours.

47 We therefore adopted this method for the synthesis of 48 nanoparticles 4 . Coupling between 3 and 5 was performed 49 with HATU and DIPEA in $\mathrm{CH}_{2} \mathrm{Cl}_{2}$, affording the desired 50 nanoparticles 4. We found this method both safe and 51 operationally simple for many reasons. Firstly, HATU is an 52 established chemical which is widely used during large scale 53 synthesis of pharmaceuticals. Several reports of HATU 54 applications have showcased its use in the synthesis of 55 promising drug candidates (Dunetz et al. 2016 and references

56 cited herein). Secondly, a simple filtration/washing cycle can 57 be used to purify and isolate nanoparticles 4 . Thirdly, the 58 reaction also proceeded both at room temperature and in 59 presence of relatively safe chemicals. Finally, our approach is 60 highly reproducible. As a proof, we repeated the synthesis 61 three times, obtaining nanoparticles 4 with the same zeta 62 potentials in all cases (see Table 1). Yields of nanoparticles 4 63 were $91 \%, 88 \%$ and $90 \%$ for repetitions $1-3$ respectively. We 64 therefore believe that our approach to nanoparticles 4 can be 65 feasible for a scale-up production. The tetrazine grafted on 66 IONP render a nano-platform for conjugating a diverse range 67 of biomolecules or drugs via copper-free click chemistry. 68 This strategy has advantages of simplifying chemical 69 synthesis, avoiding cytotoxic copper catalysts and generating 70 a system to detect biomarkers in live cells with improved 71 sensitivity. One such example is the nanoparticle-antibody 72 conjugate developed to image extracellular receptors in 73 cancer cells (Haun et al. 2010).

\section{4}

75 Table 1. Zeta potentials found for nanoparticles precursor 3, 76 tetrazine bound IONPs 4 and yields obtained.

77

78

\begin{tabular}{ccc}
\hline $\begin{array}{c}\text { Sample } \\
\text { (repeat) }\end{array}$ & $\begin{array}{c}\text { IONPs 3 } \\
(\mathrm{mV})\end{array}$ & $\begin{array}{c}\text { IONPs 4 } \\
(\mathrm{mV})\end{array}$ \\
\hline $\mathbf{1}$ & -37.6 & -20.5 \\
\hline $\mathbf{2}$ & -41.1 & -16.1 \\
\hline $\mathbf{3}$ & -42.9 & -18.1 \\
\hline
\end{tabular}

79 With nanoparticles 4 successfully prepared, we then decided 80 to assess their shape and average size. These parameters are 81 relevant in assessing IONPs sensitivity for biosensing 82 applications. As a proof, in 2013 Kolhatkar et al. 83 demonstrated that cubic and spherical nanoparticles display 84 different sensitivities due to their different contact areas. With 85 this in mind, we obtained TEM images of IONPs 4 (Figures $861 \mathrm{a}-\mathrm{d}$ ). It can be seen that the particles are spherical in shape 87 with a size range between 16 and $21 \mathrm{~nm}$.
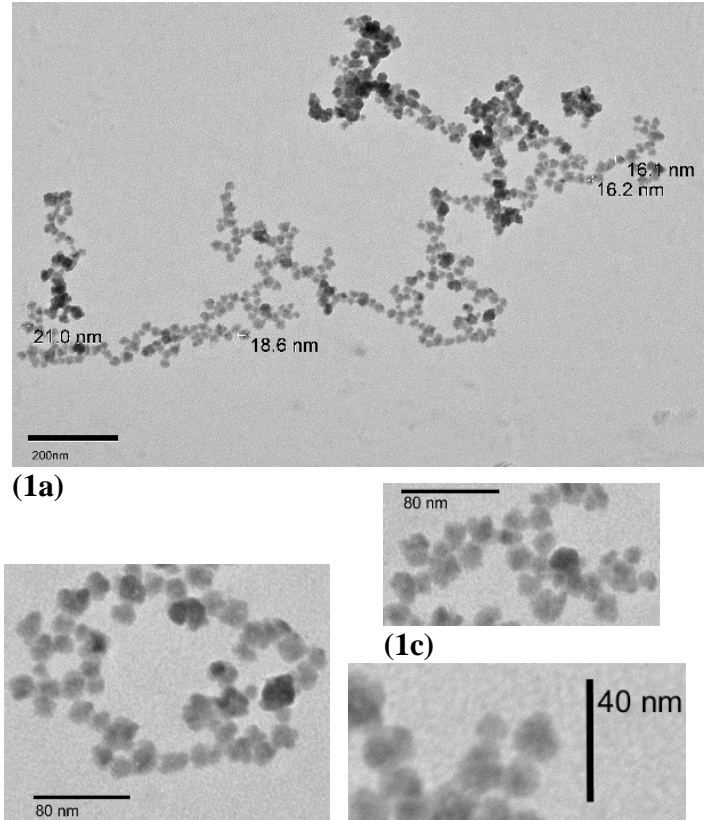

(1b)

88

89

90

91

92 Once that both shape and size of the nanoparticles were 93 assessed, we moved to establish their magnetic properties. 
$1 \mathrm{We}$ were particularly interested in the saturation 2 magnetization value because this is another key indicator in 3 assessing IONPs sensitivity for biosensing applications 4 (Colombo et al. 2012).

5 Magnetization curves were performed on a solid sample. 6 IONPs 4 showed a paramagnetic behaviour with a saturation 7 magnetization value of 1.49E-03 emu/g (Figure 2), 8 confirming also that IONPs 4 present a degree of surface 9 functionalisation.

11 Fig 2. Magnetization curve of IONPs 4 at 310K

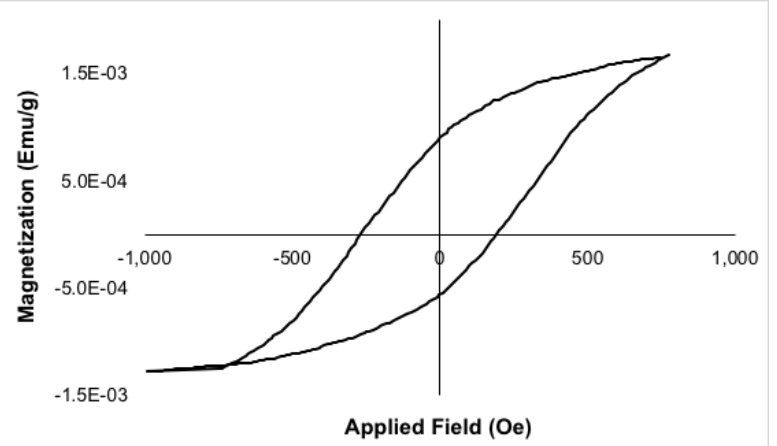

12

13 To further demonstrate the potential applicability of 14 nanoparticles 4 in pre-clinical and clinical fields, we felt 15 mandatory to perform toxicological screenings. After being 16 cultured in the presence of nanoparticles 4 at various 17 concentrations for one to three days, both metabolic activity 18 (Figure 3) and total DNA of HUVECs (Figure 4) were 19 performed, with no cytotoxicity or changes in cell viability 20 and morphology encountered.

21

22 Fig. 3 Metabolic profiles of HUVEC cells treated at different 23 concentrations of IONPs 4.

24

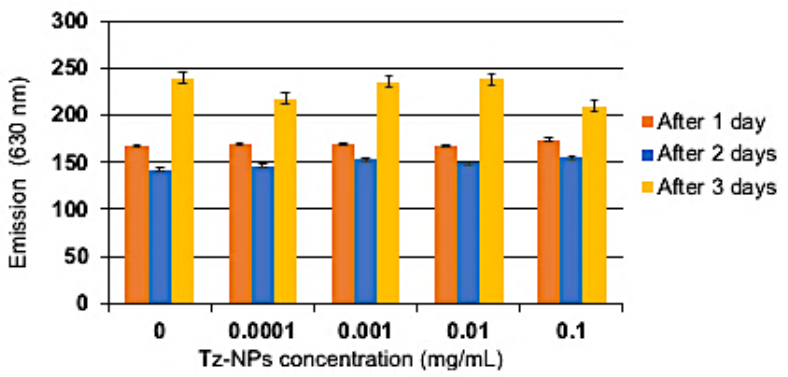

25 Fig. 4 Total DNA of HUVEC cells treated at different 27 concentrations of IONPs 4.

28

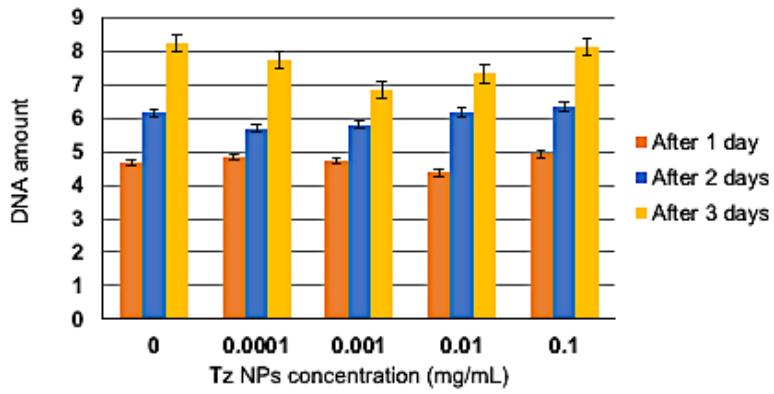

31 These results strongly suggest that targeted contrast reagents 32 based on IONPs $\mathbf{4}$ can be safely used in pre-clinical and 33 clinical imaging or magnetic-nanomedicine.

34 The relaxation rate is another key parameter in the 35 applicability of nanoparticles 4 in preclinical and clinical
36 fields. We plan to obtain this parameter as a part of our future 37 applications in vivo, and the results will be reported in due 38 course.

39

\section{Conclusions}

41

42 We have developed an operationally simple, high-yielding 43 and cost-effective protocol to produce a novel class of low 44 toxicity, tetrazine-based IONPs. Our protocol is reproducible 45 and the tetrazine motif can be exploited for the preparation of 46 conjugated biomolecules or drug molecules for targeted47 based contrast agents or nanomedicine by using all the 48 strengths of the well-known copper-free click chemistry. 49 Targeted magnetic nanoparticle-based contrast agents and 50 nanomedicines are rapidly emerging into the preclinical and 51 clinical fields. We therefore anticipate that our method can 52 represent an invaluable breakthrough and provides a multi53 functional nanoparticle platform for the large-scale 54 production and commercialization of improved, tissue 55 specific contrast agents or magnetic nanomedicine for the 56 clinical diagnosis and therapy of diseases at an asymptomatic 57 stage.

58

\section{Conflicts of interest}

60

61 None to declare

62

63 Acknowledgements

64

65 This work is supported by the British Heart Foundation 66 [FS/15/17/31411 to M. M. M.].

\section{7}

\section{References}

69

70 Baskin JM, Prescher JA, Laughlin ST, Agard NJ, Chang PV, 71 Miller IA, Lo A, Codelli JA, Bertozzi CR (2007).

72 Copper-free click chemistry for dynamic in vivo 73 imaging. Proc Natl Acad Sci USA 104: 1679374 16797. doi: 10.1073/pnas.0707090104

75 Colombo M, Carregal-Romero S, Casula MF, Gutierrez L, 76 Morales MP, Bohm IB, Heverhagen JT, Prosperi D, 77 Parak WJ (2012) Biological applications of 78 magnetic nanoparticles Chem Soc Rev 41:4306794334 https://doi:10.1039/c2cs15337h

80 Debets MF, van Berkel SS, Schoffelen S, Rutjes FP, van Hest 81 JC, van Delft FL (2010) Aza-dibenzocyclooctynes 82 for fast and efficient enzyme PEGylation via copper83 free $(3+2)$ cycloaddition ChemComm 46:97-99 84 https://doi:10.1039/b917797c

85 Devaraj NK, Weissleder R (2011) Biomedical Applications

86 of Tetrazine Cycloadditions Acc Chem Res 44:816-

87827 https://doi:10.1021/ar200037t

88 Dunetz JR, Magano J, Weisenburger GA (2016) Large-Scale 89 Applications of Amide Coupling Reagents for the 90 Synthesis of Pharmaceuticals Org Process Res Dev 91 20:140-177 https://doi:10.1021/op500305s

92 Evans HL, Nguyen QD, Carroll LS, Kaliszczak M, Twyman 93 FJ, Spivey AC, Aboagye EO (2014) A 94 bioorthogonal (68) Ga-labelling strategy for rapid in 95 vivo imaging ChemComm 50:9557-9560 96 https://doi:10.1039/c4cc03903c

97 Haun JB, Devaraj NK, Hilderbrand SA, Lee H, Weissleder R 98 (2010) Bioorthogonal chemistry amplifies 99 nanoparticle binding and enhances the sensitivity of 
1 cell detection Nature Nanotechnonogy 5:660-665 2 https://doi:10.1038/nnano.2010.148

3 Herranz F, Morales MP, Roca AG, Vilar R, Ruiz-Cabello J 4 (2008) A new method for the aqueous 5 functionalization of superparamagnetic $\mathrm{Fe}_{2} \mathrm{O}_{3}$ 6 nanoparticles Contrast Media Mol Imaging 3:2157222 https://doi:10.1002/cmmi.254

8 Jaradat DMM (2018) Thirteen decades of peptide synthesis: 9 key developments in solid phase peptide synthesis 10 and amide bond formation utilized in peptide 11 ligation Amino Acids 50:39-68

13 Kolhatkar AG, Nekrashevich I, Litvinov D, Willson RC, Lee 14 TR (2013). Cubic Silica-Coated and Amine15 Functionalized FeCo Nanoparticles with High 16 Saturation Magnetization. Chem Mater, 25:1092171097 https://doi: 10.1021/cm304111z

18 Kuzmin A, Poloukhtine A, Wolfert MA, Popik VV (2010)

22 McKay CS, Finn MG (2014) Click chemistry in complex 23 mixtures: bioorthogonal bioconjugation Chem Biol $24 \quad 21: 1075-1101$

25 https://doi:10.1016/j.chembiol.2014.09.002

26 Meloni MM, Barton S, Xu L, Kaski JC, Song W, He T (2017)

27 Contrast agents for cardiovascular magnetic 28 resonance imaging: an overview J Mater Chem B 30 Mishra SK, Kumar BS, Khushu S, Tripathi RP, Gangenahalli 31
36 Morawski AM, Lanza GA, Wickline SA (2005) Targeted 37 contrast agents for magnetic resonance imaging and 38 ultrasound Curr Opin Biotechnol 16:89-92 39 https://doi:10.1016/j.copbio.2004.11.001

40 Ohgushi M, Nagayama K, Wada A (1978) Dextran41 magnetite: A new relaxation reagent and its 42 application to T2 measurements in gel systems $\mathrm{J}$ 43 Magn Reson (1969) 29:599-601 44 https://doi.org/10.1016/0022-2364(78)90018-5

45 Oliveira BL, Guo Z, Bernardes GJL (2017) Inverse electron 46 demand Diels-Alder reactions in chemical biology 47 Chem Soc Rev 46:4895-4950 48 https://doi:10.1039/c7cs00184c

49 Schmitz SA, Taupitz M, Wagner S, Wolf K-J, Beyersdorff D, 50 Hamm B (2001) Magnetic resonance imaging of 51 atherosclerotic plaques using superparamagnetic 52 iron oxide particles J Magn Reson Im 14:355-361 53 https://doi:10.1002/jmri.1194

54 Trivedi RA, U-King-Im JM, Graves MJ, Kirkpatrick PJ, $55 \quad$ Gillard JH (2004) Noninvasive imaging of carotid 56 plaque inflammation Neurology 63:187-188 57 https://doi:10.1212/01.wnl.0000132962.12841.1d 